\title{
Amelioration of Behavioural, Biochemical, and Neurophysiological Deficits by Combination of Monosodium Glutamate with Resveratrol/Alpha-Lipoic Acid/Coenzyme Q10 in Rat Model of Cisplatin-Induced Peripheral Neuropathy
}

\author{
Naini Bhadri, Tejaswi Sanji, Hariprasad Madakasira Guggilla, and Rema Razdan \\ Department of Pharmacology, Al-Ameen College of Pharmacy, Bangalore, Karnataka-560027, India \\ Correspondence should be addressed to Naini Bhadri; nainipharmacology@gmail.com
}

Received 31 August 2013; Accepted 2 October 2013

Academic Editors: J.-T. Cheng and S. Wu

Copyright (C) 2013 Naini Bhadri et al. This is an open access article distributed under the Creative Commons Attribution License, which permits unrestricted use, distribution, and reproduction in any medium, provided the original work is properly cited.

Cisplatin or cis-diamminedichloroplatinum (II) (CDDP) is a cytotoxic chemotherapeutic agent with dose-dependent peripheral neuropathy as a foremost side effect characterised by ataxia, pain, and sensory impairment. Cumulative drug therapy of CDDP is known to produce severe oxidative damage. It mainly targets and accumulates in dorsal root ganglia that in turn cause damage resulting in secondary nerve fibre axonopathy. In the present study, we investigated the neuroprotective effect of the combination of monosodium glutamate (MSG) with three individual antioxidants, that is, resveratrol, alpha-lipoic acid (ALA), and coenzyme Q10 (CoQ10), in cisplatin ( $2 \mathrm{mg} / \mathrm{kg}$ i.p. twice weekly) induced peripheral neuropathy in rats. After 8 weeks of treatment the degree of neuroprotection was determined by measuring behavioral and electrophysiological properties and sciatic nerve lipid peroxidation, as well as glutathione and catalase levels. The results suggested that pretreatment with the combination of MSG (500 mg/kg/day po) with resveratrol (10 mg/kg/day i.p.) or ALA (20 mg/kg/day i.p.) or CoQ10 (10 mg/kg weekly thrice i.p.) exhibited neuroprotective effect. The maximum neuroprotection of MSG was observed in the combination with resveratrol.

\section{Introduction}

Cisplatin is an effective antineoplastic drug widely used in the treatment of malignancies including ovarian, lung, oesophagus, stomach, bladder, and testicular cancers. However, its use is limited by its serious adverse effects including peripheral neuropathy and nephrotoxicity [1]. The reported incidence of neuropathy varies between $24 \%$ and $92 \%$ [2]. Characteristic features of cisplatin neurotoxicity are mainly ataxia, pain, and sensory impairment. Symptoms are prominent in patients receiving cumulative doses of cisplatin above $300 \mathrm{mg} / \mathrm{m}^{2}$ or $500 \mathrm{mg} / \mathrm{m}^{2}$ [3]. About $20 \%$ of patients have shown poor compliance for the course of cisplatin therapy attributable to sensory neuropathy [4]. The mechanism of cisplatin-induced neuropathy is not completely understood; it is preferentially taken up in to dorsal root ganglia and is believed to affect the stem of the nerves leading to loss of sensory neurons affecting large myelinated sensory nerve fibers [5]. However, data obtained in human studies indicates that cisplatin treatment induces a fall in plasma antioxidant level because of oxidative stress [6] several mechanism including hypoxia, inflammation and free radicals induced apoptosis are thought to be involved. Excessive production of free radicals, such as superoxide anion, hydrogen peroxide, and hydroxyl radicals, and the occurrence of lipid per oxidation due to oxidative stress are associated with cisplatin-induced neuropathy.

Resveratrol (3,5, $4^{\prime}$-trihydroxystilbene), a naturally occurring polyphenol found in grapes and red wine, has been reported to have excellent antioxidant activity. It not only prevents free radicals formation but also attenuates their toxicity by inhibiting the lipid per oxidation [7]. It has shown to exert neuroprotective, cancer chemopreventive, anti-inflammatory, and cardioprotective activities [8].

Coenzyme Q10 (CoQ10) or Ubiquinone is an oil-soluble, vitamin-like substance present in almost all eukaryotic cells, primarily in the mitochondria. It is a component of the electron transport chain and participates in aerobic cellular respiration, generating energy in the form of ATP [9]. In recent 
years research also focused on its antioxidant properties in several cellular compartments [10]. CoQ10 has shown protective effect against neuronal loss in several animal models of neurodegeneration and the ability of CoQ10 to slow disease progression in clinical trials has been considered to explore neuroprotective activity in many of the neurodegenerative diseases [11].

Alpha-lipoic acid or 1,2-dithiolane-3-pentanoic acid (ALA) is a naturally available dithiol compound synthesized enzymatically in the mitochondrion from octanoic acid [12]. ALA reacts with reactive oxygen species such as superoxide radicals, hydroxyl radicals, hypochlorous acid, peroxyl radicals, and singlet oxygen. Administration of ALA has been shown to be beneficial in several oxidative stress models such as ischemia-reperfusion injury, diabetes, cataract formation, HIV activation, neurodegeneration, and radiation injury [13].

Monosodium glutamate (MSG) is the sodium salt of glutamic acid. It is widely used as food additive mainly as flavour enhancer and it is believed to be safe without serious concerns. It contributes an important role in the biosynthesis of several key amino acids [14]. Evidence from clinical and preclinical studies revealed that it is a major oxidative fuel for the gut and that dietary glutamate extensively undergoes first pass metabolism by the intestine. It is an important precursor for synthesis of bioactive molecules, including glutathione, an endogenous antioxidant, and also functions as a key neurotransmitter [15]. Partial investigation of MSG in clinical trial as a neuroprotectant in the early 1980s has shown efficacy in preventing neuropathy induced by vincristine [16]. A new class of glutamate receptors, named metabotropic glutamate receptors (mGluRs) that are coupled to effector systems through GTP proteins (G-proteins), became evident by the majority of most recent research activities. Activation of (mGluRs) subtypes usually regulates many cellular activities in mammalian systems in various experimental models. Activation of (mGluRs) subtypes protected cells against various types of neuronal insults such as traumatic brain injury, stroke, and NMDA excitotoxicity [17]. Interestingly, in recent years, glutamate has been reported to be efficient in preventing neuropathy caused by many cytotoxic drugs in mice, rats, and humans with no or minimal apparent intrinsic toxicity and its low cost and oral availability make it attractive for clinical use as a neuroprotectant [18].

In the present study we further explored its potential in rodents for its neuroprotective activity in presence of other antioxidants, namely, resveratrol, coenzyme Q10, and ALA, in rat model of cisplatin-induced peripheral neuropathy by assessing behavioral, biochemical, and neurophysiological parameters.

\section{Materials and Methods}

2.1. Reagents. All reagents and solvents used were of analytical grade. Cisplatin ( $2 \mathrm{mg} / \mathrm{kg}$ i.p. twice weekly) was used to induce peripheral neuropathy by intraperitoneal injection [19]. Aqueous solution of MSG (Himadia Labs, India) was used at $500 \mathrm{mg} / \mathrm{kg} /$ day p.o. dose [18]. Resveratrol (Sami Labs, India) solution was freshly prepared using $2 \% \mathrm{v} / \mathrm{v}$ ethanol at a dose of $10 \mathrm{mg} / \mathrm{kg} /$ day i.p. [7]. Suspension of ALA (Sami
Labs, India) in sterile saline and $\mathrm{NaOH}$ was used at a dose $25 \mathrm{mg} / \mathrm{kg} /$ day i.p. [20]. Solution of coenzyme Q10 (Himedia Labs, India) was dissolved in $2 \% \mathrm{v} / \mathrm{v}$ ethanol and was used at a dose of $10 \mathrm{mg} / \mathrm{kg}$ i.p./day [21]. All solutions were freshly prepared every day before dosing the animals.

2.2. Animals. Animal procedures were done in accordance with the Institutional Animal Ethics Committee of CPCSEA. Adult Wistar rats (175-225 g) of both sexes were housed in an aseptic animal room at a temperature of $20-24^{\circ} \mathrm{C}$ and a humidity of $40-70 \%$, with a $12 \mathrm{~h}$ light cycle and 12 fresh air changes per hour in clean paddy husk bedding. All animals were allowed free access to rat chow and water. They were acclimatized for a minimum period of 1 week prior to the beginning of the study.

2.3. Induction of Peripheral Neuropathy. Cisplatin $(2 \mathrm{mg} / \mathrm{kg}$, i.p. twice weekly) was given for 8 weeks to induce peripheral neuropathy in rats [19]. The dose of cisplatin was decided by the standardization study done previously.

2.4. Experimental Design. The animals were divided into five groups ( $n=6$ per group): normal control group (Group 1), cisplatin-induced peripheral neuropathic rats (Group 2), MSG (500 mg/kg/day p.o.) + cisplatin + resveratrol (10 mg/ kg/day i.p.) (Group 3), MSG (500 mg/kg/day p.o.) + cisplatin + CoQ10 (10 mg/kg/day i.p.) (Group 4), and MSG (500 mg/ $\mathrm{kg} /$ day p.o.) + cisplatin + ALA (25 mg/kg/day i.p.) (Group 5) daily for 8 weeks. All parameters under behavioral studies, biochemical estimation, and electrophysiology were evaluated on the completion of the treatment (Figure 1).

2.5. Assessment of General Toxicity. Body weight was measured before each administration of cisplatin or vehicle and after administration of drug combination. All rats were examined daily for clinical signs such as piloerection or hind limb weakness and to assess general health.

\subsection{Behavioral Studies}

2.6.1. Thermal Hyperalgesia. Thermal hyperalgesia was assessed by tail immersion test. It was noted with the immersion of terminal part of the tail $(1 \mathrm{~cm})$ in water maintained at $50^{\circ} \mathrm{C}$. The duration of tail withdrawal reflex or signs of struggle were recorded as response of heat sensation and a cutoff time of $20 \mathrm{~s}$ was maintained [22]. Rats were acclimatized to the testing procedure and handled by the investigator during the week before the experiment.

2.6.2. Grip Strength Assays. Grip strength meter was used for evaluating grip strength of animals. Before commencement of experiment the animals were acclimatized by placing them on the instrument for few minutes. Rats were held by the tail above the grid of grip strength meter to an almost horizontal position. The base of the tail was then pulled following the axle of the sensor until it released the grid. The force achieved by the animal was then displayed on the screen and was recorded as newtons or kg units [23]. 


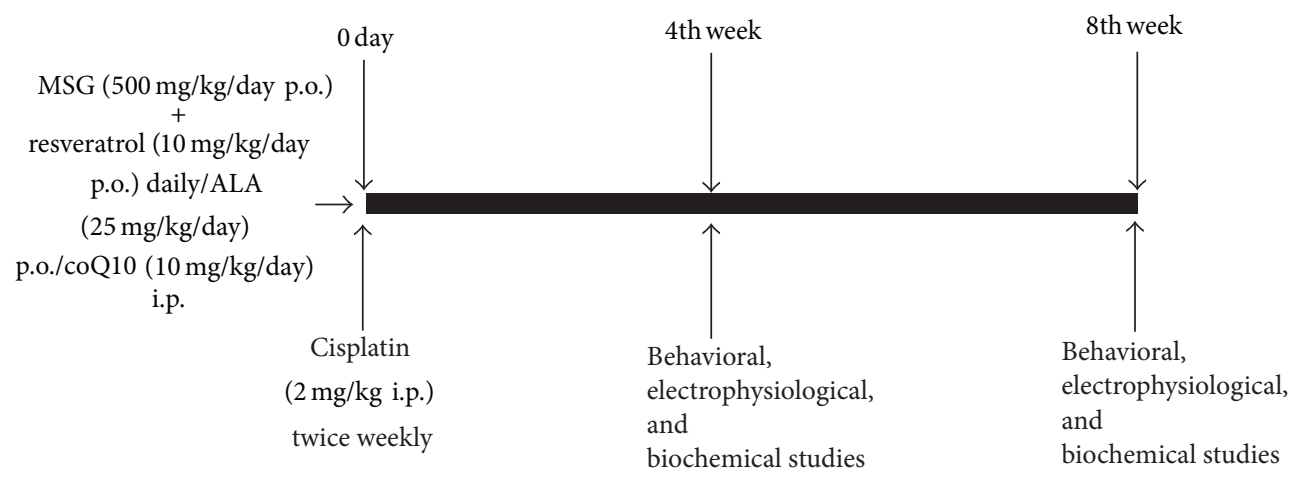

FIGURE 1: Schematic representation of experimental design.

2.6.3. Rota-Rod Performance Assessment. The rota-rod test is widely used in rodents to assess their "minimal neurological defect" such as impaired motor function (e.g., ataxia) and coordination. The rota-rod unit consists of a rotating rod, $75 \mathrm{~mm}$ in diameter, which was divided into four parts by compartmentalization to permit the testing of four rats at a time. Briefly, in a training session, the rats were placed on the rod that was set to $25 \mathrm{rpm}$ and the performance time that each rat was able to remain on the rota-rod was recorded. The rats were subjected to three training trials from $3 \mathrm{~h}$ to $4 \mathrm{~h}$ intervals on two separate days for acclimatization purposes. In the test session, the rats were placed on the rota-rod and their performance times were recorded [1].

\subsection{Electrophysiology}

2.7.1. Isolation of Sciatic Nerve. Rats were sacrificed by overdose of ketamine/xylazine i.p. Rat's back was shaved and incision was made at L4-L6 spinal segments. The sciatic nerves were surgically exposed from sciatic notch to the gastrocnemius tendon and removed, carefully impregnated on fine filter paper to remove any accompanying blood and soaked for 10 minutes in Ringer-Locke buffer to prevent spontaneous firing of the nerve [24].

2.7.2. Measurement of Nerve Conduction Velocity (NCV). Rat sciatic nerve having a length of $3 \mathrm{~cm}-5 \mathrm{~cm}$ was prepared and mounted into the nerve chamber consisting of small quantity of phosphate buffer $\mathrm{pH} 7$ to humidify the compartment. First pair of $5 \mathrm{~mm}$ spaced electrodes is connected via the stimulator cable (MLA 270) to output 1 and 2 of the power lab unit. The recording cable (MLA 285) was connected to the input 1 of the power lab and microhooks of the same cable to the desired electrode wires depending upon the length of the sciatic nerve. After recording a set of responses latency was calculated using lab chart 7 software. Distance between the stimulating electrode and recording electrode was measured. Nerve conduction velocity $(\mathrm{m} / \mathrm{sec})$ was calculated using the following formula:

$$
\mathrm{NCV}=\text { Distance }(\mathrm{mm}) / \text { latency. }
$$

\subsection{Biochemical Estimation}

2.8.1. Sciatic Nerve Homogenate Preparation. Sciatic nerve samples were rinsed with ice cold saline homogenized in chilled phosphate buffer ( $\mathrm{pH}$ 7.4) and used for the estimation of lipid per oxidation (MDA levels), reduced glutathione and catalase [25].

2.8.2. Estimation of Lipid per Oxidation. The extent of lipid per oxidation in terms of thiobarbituric acid reactive substances (TBARS) formation was measured according to the method of Esterbauer and Cheeseman. Tissue extracts were mixed separately with $1 \mathrm{~mL}$ TCA $(20 \%)$ and $2 \mathrm{~mL}$ TBA $(0.67 \%)$ and heated for $1 \mathrm{~h}$ at $100^{\circ} \mathrm{C}$. After cooling, the precipitate was removed by centrifugation. The absorbance of each sample was measured at $535 \mathrm{~nm}$ using a blank containing all the reagents except the sample. As 99\% TBARS are malondialdehyde (MDA), therefore TBARS concentrations of the samples were calculated using the extinction coefficient of MDA, that is, $1.56 \times 10^{5} \mathrm{M}^{-1} \mathrm{~cm}^{-1}$, and were expressed as $\mu \mathrm{M}$ of malondialdehyde per mg protein [26].

2.8.3. Estimation of Reduced Glutathione. Reduced glutathione was assayed by the method of [27]. Briefly $1.0 \mathrm{~mL}$ of sciatic nerve homogenate $(10 \% \mathrm{w} / \mathrm{v})$ was precipitated with $1.0 \mathrm{~mL}$ of sulphosalicylic acid (4\%). The samples were kept at $40^{\circ} \mathrm{C}$ for at least $1 \mathrm{~h}$ and then subjected to centrifugation at $1200 \mathrm{~g}$ for $15 \mathrm{~min}$ at $40^{\circ} \mathrm{C}$. The assay mixture contained $0.1 \mathrm{~mL}$ supernatant, $2.7 \mathrm{~mL}$ phosphate buffer $(0.1 \mathrm{M}, \mathrm{pH} 7.4)$, and $0.2 \mathrm{~mL} 5,5$,dithiobis (2-nitro benzoic acid) (Ellman's reagent, $0.1 \mathrm{mM}, \mathrm{pH} 8.0$ ) in a total volume of $3.0 \mathrm{~mL}$. The yellow colour developed was read immediately at $412 \mathrm{~nm}$ and the reduced GSH levels were expressed as $\mu \mathrm{g} / \mathrm{mg}$ protein.

2.8.4. Estimation of Catalase. Catalase was estimated by the method of Aebi [28] which is a quantitative spectroscopic method developed for following the breakdown of $\mathrm{H}_{2} \mathrm{O}_{2}$ at $240 \mathrm{~nm}$ in unit time. The sample readings were taken by placing $1 \mathrm{~mL}$ of phosphate buffer and $100 \mu \mathrm{L}$ of tissue homogenate in the reference cuvette and $1 \mathrm{~mL}$ of $\mathrm{H}_{2} \mathrm{O}_{2}$ and $100 \mu \mathrm{L}$ of homogenate in the test cuvette in the spectrophotometer. For 
each measurement, the reading was taken at $240 \mathrm{~nm} 1 \mathrm{~min}$ after placing the cuvettes in Shimadzu spectrophotometer.

2.9. Statistical Analysis. Results were expressed as mean \pm S.E.M. The intergroup variation was measured by one-way analysis of variance (ANOVA) followed by Tukey's multiple comparison test. The statistical analysis was done using the Graph pad prism software version 5.0. Probability values $P<$ 0.05 were considered to be significant.

\section{Results}

3.1. Assessment of General Toxicity. No deterioration in general status was observed; no alterations in the body temperature and no abnormal clinical signs were observed. No rats in the cisplatin control group died during the course of the experiment. Cisplatin treated rats (b.w. $186 \pm 1.55 \mathrm{~g}$ ) showed significant reduction in body weight as compared with the control group (b.w. $216 \pm 1.78 \mathrm{~g}$ ). Treatment with a combination of MSG + resveratrol (b.w. $202 \pm 2.55$ g), MSG + coenzyme Q10 (b.w. $205 \pm 2.9$ g), and MSG + ALA (b.w. 204 $\pm 3.32 \mathrm{~g}$ ) did not produce any major effect on body weight of the treated rats as compared to the control group (Figure 2).

\subsection{Behavioral Testing}

3.2.1. Effect of Drug Treatment on Nociceptive Threshold in Tail Immersion (Warm Water) Test. At the end of the 4th and 8th weeks, cisplatin group exhibited significant decrease in pain threshold from noxious stimuli as compared with control rats $(P<0.01)$. MSG + resveratrol, MSG + Co Q10, and MSG + ALA administration for 8 weeks significantly increased the pain threshold as compared with control neuropathic rats $(P<0.01$, Figure 3$)$.

3.2.2. Modulation of Muscle Hyperalgesia Using Grip Strength Assay. Administration of MSG + resveratrol, MSG + CoQ10, and MSG + ALA for 8 weeks significantly improved the latency of grip strength when compared with cisplatin treated rats. Treatment with the combination of MSG + resveratrol and MSG + ALA was the most effective $(P<0.01)$ (Figure 4$)$.

3.2.3. Modulation of Motor Coordination Using Rota-Rod Test. Cisplatin-induced neuropathy impaired motor coordination as evaluated by the walking time on a rotating rod and the number of falls (rota-rod test). Indeed, normal rats were able to balance on the rotating rod for $120.7 \pm 0.33 \mathrm{sec}$ as compared with cisplatin treated group $(P<0.001)$. Motor coordination was significantly improved after administration of the combinations of MSG + resveratrol, MSG + CoQ10, and MSG + ALA when compared with cisplatin treated rats. Combination of MSG + resveratrol treatment was the most effective $(P<0.05)$ (Figure 5).

3.3. Effect of Cisplatin on Nerve Conduction Velocity. Eight weeks after neuropathy, neuropathic rats showed significant decrease in NCV as compared with the normal group. Treatment with combination of MSG + resveratrol, MSG + CoQ10, and MSG + ALA reversed the nerve conduction

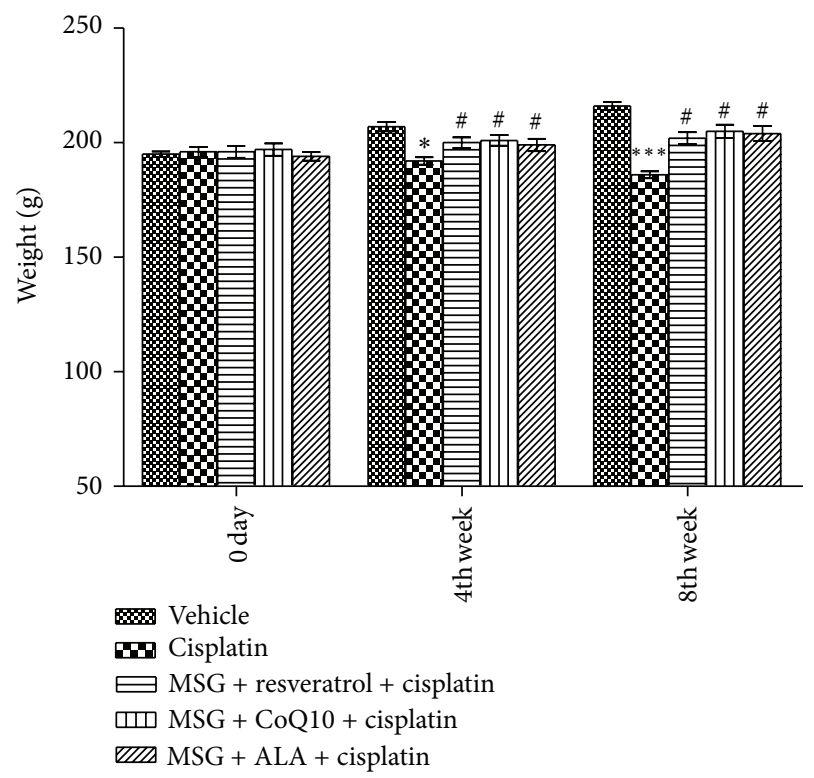

FIGURE 2: Effect of chronic treatment with combination of MSG and resveratrol/ALA/Co Q10 on body weight. ${ }^{*}(P<0.05),{ }^{* * *}(P<$ $0.001)$ versus control group, ${ }^{\#}(P<0.05)$ versus cisplatin treated group.

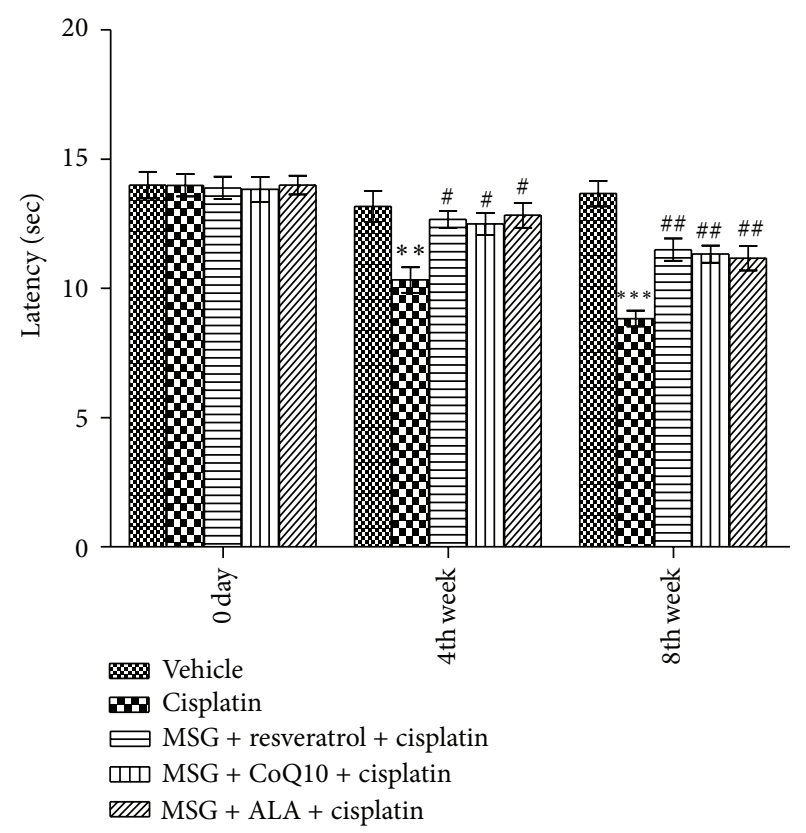

FIGURE 3: Effect of chronic treatment with combination of MSG and resveratrol/ALA/Co Q10 on thermal hyperalgesia in cisplatin treated rats. ${ }^{* *}(P<0.001),{ }^{* * *}(P<0.001)$ versus control group, ${ }^{\#}(P<$ $0.05),{ }^{\# \#}(P<0.01)$ versus cisplatin treated group.

in neuropathic rats and significantly improved nerve conduction velocity when compared with cisplatin treated rats. MSG + resveratrol and MSG + ALA treatments were the most effective (Figure 6). 


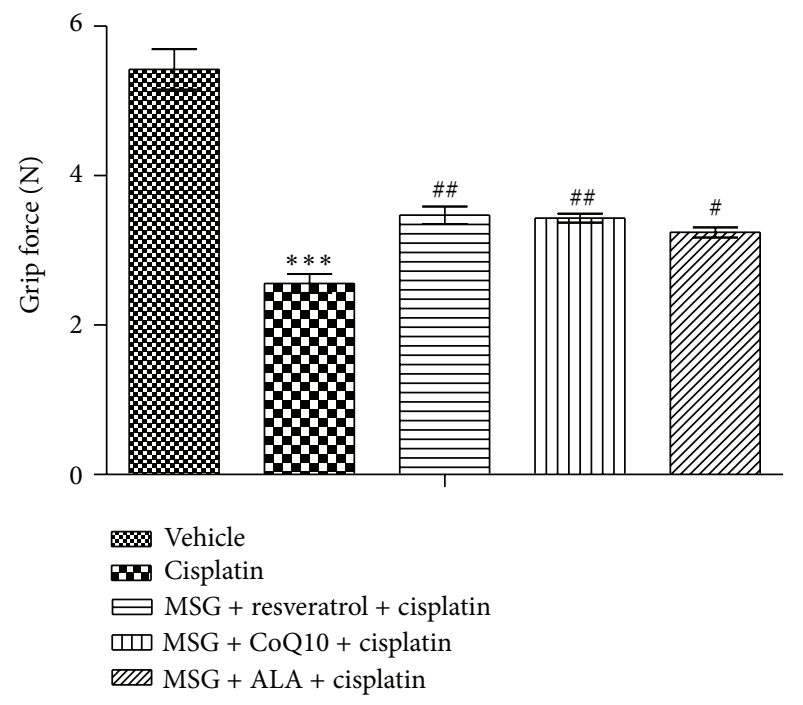

FIGURE 4: Effect of chronic treatment with combination of MSG and resveratrol/ALA/Co Q10 on grip strength in cisplatin treated rats. ${ }^{* * *}(P<0.001)$ versus control group, ${ }^{\#}(P<0.05),{ }^{\# \#}(P<0.01)$ versus cisplatin treated group.

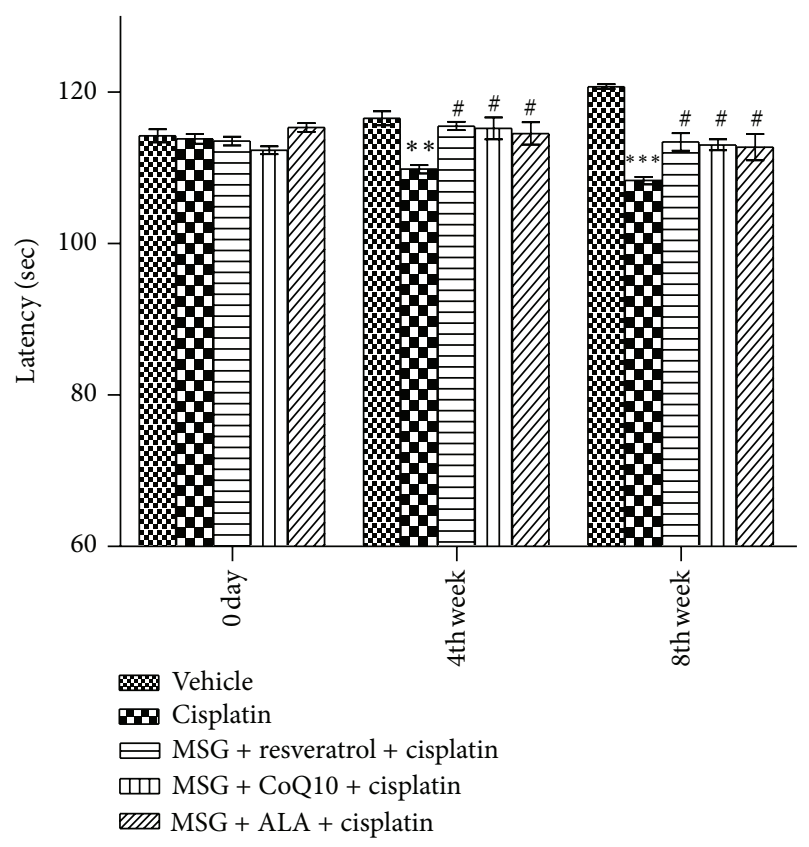

FIGURE 5: Effect of chronic treatment with combination of MSG and resveratrol/ALA/Co Q10 on motor coordination in cisplatin treated rats. ${ }^{* *}(P<0.01),{ }^{* * *}(P<0.001)$ versus control group, ${ }^{\#}(P<0.05)$, versus cisplatin treated group.

\subsection{Biochemical Estimation}

3.4.1. Effect of Cisplatin-Induced Changes on Lipid per Oxidation. After administration of cisplatin, neuropathic rats showed significant increase in MDA levels in sciatic nerve as compared with the normal group. Treatment with the combination of MSG + resveratrol, MSG + CoQ10, and MSG + ALA at the 4th and 8th weeks showed a significant decrease when

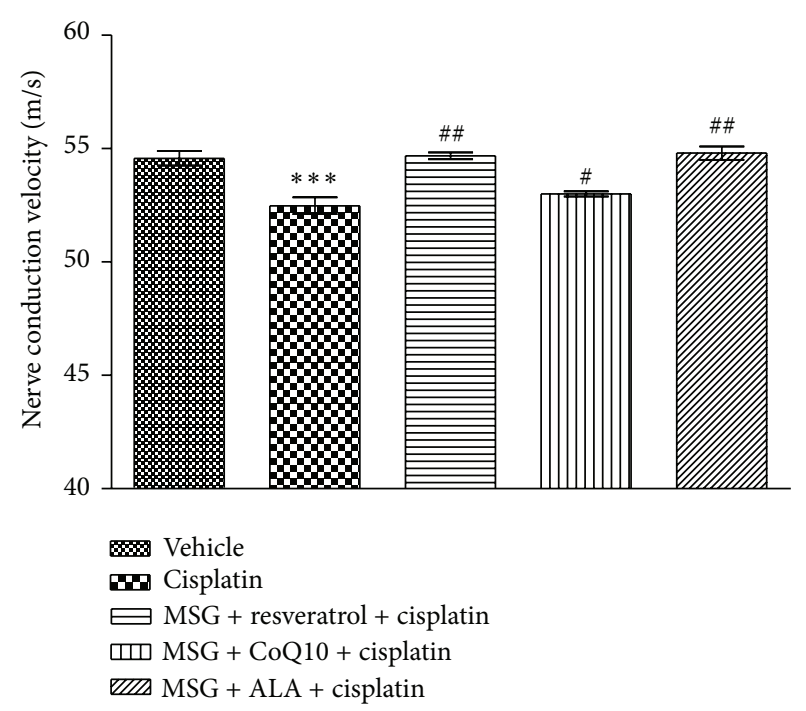

FIGURE 6: Effect of chronic treatment with combination of MSG and resveratrol/ALA/Co Q10 on nerve conduction velocity in sciatic nerve of cisplatin treated rats. ${ }^{* * *}(P<0.001)$ versus control group, ${ }^{\#}(P<0.05),{ }^{\# \#}(P<0.01)$ versus cisplatin treated group.

compared with cisplatin treated group. MSG + resveratrol treatment was the most effective in reducing the MDA level (Table 1).

3.4.2. Effect of Cisplatin on Reduced Glutathione. Neuropathic rats showed significant decrease in GSH levels in the sciatic nerve as compared with normal group. Treatment with the combination of MSG + resveratrol, MSG + CoQ10, and MSG + ALA showed a significance increase in GSH level. Combination of MSG + resveratrol was the most effective among all combinations (Table 1).

\subsubsection{Effect of Cisplatin-Induced Changes on Catalase Activity.} Sciatic nerve catalase activity was significantly increased in the 8 th week after administration of the combinations of MSG + resveratrol, MSG + CoQ10, and MSG + ALA as compared to cisplatin treated rats in which a lower level of catalase activity was observed (Table 1).

\section{Discussion}

Although cisplatin is an effective antineoplastic drug, its use is limited by its serious adverse effects including peripheral neuropathy and nephrotoxicity [1]. It exhibits its toxic effects on cell by inducing oxidative stress and loss of sensory neurons. In this study the model of cisplatin-induced peripheral neuropathy is used to assess the effectiveness of the few putative combinations of neuroprotective agents, namely, glutamate, resveratrol, ALA, and CoQ10. Rat model is used as it is qualitatively similar to that of humans. It is reported that cisplatin predominantly accumulated in the dorsal root ganglia causing nucleolar damage and also affecting Schwann cells which play a vital role in nerve development and regeneration there by signalling apoptosis [29]. Cisplatin has also been 
TABLE 1: Effect of chronic treatment with combination of MSG and resveratrol/ALA/Co Q10 on levels of MDA (a marker of lipid peroxidation), reduced glutathion, and catalase in sciatic nerve of cisplatin treated rats.

\begin{tabular}{lcccc}
\hline SL. NO & Group & $\begin{array}{c}\text { MDA } \\
(\mu \mathrm{M} / \mathrm{mg} \text { protein })\end{array}$ & $\begin{array}{c}\text { GSH } \\
(\mu \mathrm{g} / \mathrm{mg} \text { protein })\end{array}$ & $\begin{array}{c}\text { Catalase } \\
(\mathrm{U} / \mathrm{mg} \text { protein })\end{array}$ \\
\hline 1 & Vehicle & $1.52 \pm 0.09$ & $70.34 \pm 0.33$ & $0.106 \pm 0.010$ \\
2 & Cisplatin & $2.76 \pm 0.19^{* * *}$ & $57.66 \pm 0.69^{* * *}$ & $0.051 \pm 0.004^{* * *}$ \\
3 & Cisplatin + MSG + Res & $2.12 \pm 0.08^{\# \#}$ & $61.72 \pm 1.19^{\# \#}$ & $0.091 \pm 0.006^{\# \#}$ \\
4 & Cisplatin + MSG + Co Q10 & $2.22 \pm 0.04^{\#}$ & $60.63 \pm 0.47^{\#}$ & $0.088 \pm 0.008^{\#}$ \\
5 & Cisplatin + MSG + ALA & $2.32 \pm 0.02^{\#}$ & $60.76 \pm 0.49^{\#}$ & $0.095 \pm 0.010^{\# \#}$ \\
\hline
\end{tabular}

${ }^{* * * *}(P<0.001)$ versus control group, ${ }^{\#}(P<0.05),{ }^{\# \#}(P<0.01)$ versus cisplatin treated group.

reported to cause ROS generation and causes lipid per oxidation. Recent pieces of evidence suggest that cisplatin reduces peripheral nerve blood supply through potent angiogenic effect which may predispose the nerve damage [3]. Significant loss of body weight, motor coordination abnormality, and a decrease of muscle power could be observed at the 4th week and kept on progressively increasing till the end of the study (8th week). These parameters appeared to be sensitive and reliable to demonstrate neuropathic conditions. Reduction in nerve conduction velocity at the end of treatment confirmed abnormal functions of nerve. Thus, peripheral neuropathy was successfully induced in rats and this model can be used for the screening of potential neuroprotective drugs for the prevention of cisplatin-induced peripheral neuropathy.

In the present study treatment with a combination of MSG $(500 \mathrm{mg} / \mathrm{kg} /$ day p.o.) with resveratrol $(10 \mathrm{mg} / \mathrm{kg} /$ day i.p.)/ALA (20 mg/kg/day i.p.)/Co Q10 (10 mg/kg/day i.p.) significantly alleviated cisplatin-induced peripheral neuropathy in rats. Rats treated with the combination exhibited less decrease in body weight, decreased thermal hyperalgesia, improved motor coordination, increased grip strength, improved nerve conduction velocity, and higher levels of antioxidant enzymes compared with cisplatin treated rats.

Resveratrol has been shown to have significant antioxidant and anti-inflammatory properties and found to be a neuroprotective agent against excitotoxicity [30] Reports suggest that resveratrol corrects nerve blood flow deficits due to direct vasodilatation and it has already been shown to produce vasodilatation by both endothelial dependent and endothelium independent pathways [9]. The neuroprotective role of resveratrol in our study could probably be due to its antioxidant activity and improvements in nerve blood flow and restored motor nerve conduction velocity deficits. Nerve blood flow needs to be measured to further strengthen the mechanism of resveratrol in our study. ALA is a potent antioxidant and unlike other antioxidants it is both fat- and water-soluble which works throughout the body. ALA plays a key role in most crucial biochemical functions of mitochondria; thus, it can help with maintaining the mitochondrial membrane potential differential by controlling substrate availability for respiratory chain and regulating the redox state. Several studies provided evidence that ALA is protective in diseases characterized by mitochondrial impairment like diabetic neuropathy [31]. ALA is capable of scavenging a variety of reactive oxygen species. It scavenges hydroxyl radicals and hypochlorous acid and also terminates singlet oxygen. In our study ALA was able to improve the levels of endogenous antioxidants and decreased the lipid per oxidation. These findings could explain the neuroprotective role of ALA.

CoQ10 is a crucial component of the oxidative phosphorylation system in the mitochondria, as it is involved in oxidative phosphorylation of various products of nutrients such as fatty acids and carbohydrates where energy derived from reduced equivalents is converted into ATP to drive cellular machinery and biosynthetic processes [32]. Recent studies explored the new biochemical functions of CoQ10 including the fact that it is considered to be an essential antioxidant, regenerates other antioxidants, stimulates cell growth, and inhibits cell death. CoQ10 protects phospholipids and mitochondrial membrane proteins from per oxidation and protects DNA against oxidative damage there by functions as intracellular antioxidant [33]. In our study CoQ10 was able to reverse the cisplatin-induced oxidative damage by increasing the expression of endogenous antioxidants, preventing lipid per oxidation and thus exhibiting neuroprotective activity in cisplatin treated rats.

Glutamate is involved in the modulation of microtubule functions, which affect intracellular transportation. These processes are important for axons, which require all substances from the cell body for biosynthesis [16]. Our study shows that glutamate in combination with ALA, resveratrol, and Co Q10 has significant neuroprotective effects in cisplatin-induced neuropathy by improving endogenous antioxidant levels and probably by exerting an indirect effect on microtubules after interacting with a receptor found only on neural cells; other possible links in this pathway include local growth factor release or alteration of intracellular calmodulin and ATP which have been shown to be regulators of microtubule formation and stability [18]. However, the neuroprotective effect of glutamate can be further explained and explored by measuring the calmodulin and ATP.

\section{Conclusion}

In conclusion, we have demonstrated that MSG, resveratrol, ALA, and CoQ10 combination protected against cisplatininduced neurotoxicity by increasing endogenous antioxidants, preventing lipid per oxidation and improving NCV. Thus, these results would encourage future clinical trials of these agents in cancer patients to whom coadministration of neuroprotectants is required to prevent neurotoxicity. 


\section{Conflict of Interests}

The authors declare no competing conflict of interests.

\section{Acknowledgments}

The authors are thankful to the administration of Al-Ameen College of Pharmacy, Bangalore, India, for the financial assistance and facilities provided to the candidates.

\section{References}

[1] K. Hori, N. Ozaki, S. Suzuki, and Y. Sugiura, "Upregulations of P2X3 and ASIC3 involve in hyperalgesia induced by cisplatin administration in rats," Pain, vol. 149, no. 2, pp. 393-405, 2010.

[2] J. E. Mollman, D. J. Glover, W. M. Hogan, and R. E. Furman, "Cisplatin neuropathy: risk factors, prognosis, and protection by WR-2721," Cancer, vol. 61, no. 11, pp. 2192-2195, 1988.

[3] S. B. Park, A. V. Krishnan, C. S.-Y. Lin, D. Goldstein, M. Friedlander, and M. C. Kiernan, "Mechanisms underlying chemotherapy-induced neurotoxicity and the potential for neuroprotective strategies," Current Medicinal Chemistry, vol. 15, no. 29, pp. 3081-3094, 2008.

[4] E. S. McDonald, K. R. Randon, A. Knight, and A. J. Windebank, "Cisplatin preferentially binds to DNA in dorsal root ganglion neurons in vitro and in vivo: a potential mechanism for neurotoxicity," Neurobiology of Disease, vol. 18, no. 2, pp. 305313, 2005.

[5] A. Alaedini, Z. Xiang, H. Kim, Y.-J. Sung, and N. Latov, "Upregulation of apoptosis and regeneration genes in the dorsal root ganglia during cisplatin treatment," Experimental Neurolo$g y$, vol. 210, no. 2, pp. 368-374, 2008.

[6] A. Pace, A. Savarese, M. Picardo et al., "Neuroprotective effect of vitamin E supplementation in patients treated with cisplatin chemotherapy," Journal of Clinical Oncology, vol. 21, no. 5, pp. 927-931, 2003.

[7] A. Kumar, R. K. Kaundal, S. Iyer, and S. S. Sharma, "Effects of resveratrol on nerve functions, oxidative stress and DNA fragmentation in experimental diabetic neuropathy," Life Sciences, vol. 80, no. 13, pp. 1236-1244, 2007.

[8] H. I. Kim, T. H. Kim, and J.-H. Song, "Resveratrol inhibits Na+ currents in rat dorsal root ganglion neurons," Brain Research, vol. 1045, no. 1-2, pp. 134-141, 2005.

[9] L. Ernster and G. Dallner, "Biochemical, physiological and medical aspects of ubiquinone function," Biochimica et Biophysica Acta, vol. 1271, no. 1, pp. 195-204, 1995.

[10] G. P. Littarru and P. Langsjoen, "Coenzyme Q10 and statins: biochemical and clinical implications," Mitochondrion, vol. 7, pp. S168-S174, 2007.

[11] W. R. Galpern and M. E. Cudkowicz, "Coenzyme Q treatment of neurodegenerative diseases of aging," Mitochondrion, vol. 7, pp. S146-S153, 2007.

[12] K. P. Shay, R. F. Moreau, E. J. Smith, A. R. Smith, and T. M. Hagen, "Alpha-lipoic acid as a dietary supplement: molecular mechanisms and therapeutic potential," Biochimica et Biophysica Acta, vol. 1790, no. 10, pp. 1149-1160, 2009.

[13] L. Packer, E. H. Witt, and H. J. Tritschler, "Alpha-lipoic acid as a biological antioxidant," Free Radical Biology and Medicine, vol. 19, no. 2, pp. 227-250, 1995.

[14] B. E. Cairns, X. Dong, M. K. Mann et al., "Systemic administration of monosodium glutamate elevates intramuscular glutamate levels and sensitizes rat masseter muscle afferent fibers," Pain, vol. 132, no. 1-2, pp. 33-41, 2007.

[15] D. G. Burin and B. Stoll, "Metabolic fate and function of dietary glutamate in the gut," The American Journal of Clinical Nutrition, vol. 90, no. 3, pp. 850S-856S, 2009.

[16] F. M. Boyle, H. R. Wheeler, and G. M. Shenfield, "Amelioration of experimental cisplatin and paclitaxel neuropathy with glutamate," Journal of Neuro-Oncology, vol. 41, no. 2, pp. 107-116, 1999.

[17] M. Anjaneyulu, A. Berent-Spillson, and J. W. Russell, "Metabotropic glutamate receptors (mGluRs) and diabetic neuropathy," Current Drug Targets, vol. 9, no. 1, pp. 85-93, 2008.

[18] T. Arkaravichien, N. Sattayasai, S. Daduang, and J. Sattayasai, "Dose-dependent effects of glutamate in pyridoxine-induced neuropathy," Food and Chemical Toxicology, vol. 41, no. 10, pp. 1375-1380, 2003.

[19] R. Bianchi, M. Brines, G. Lauria et al., "Protective effect of erythropoietin and its carbamylated derivative in experimental cisplatin peripheral neurotoxicity," Clinical Cancer Research, vol. 12, no. 8, pp. 2607-2612, 2006.

[20] L. P. Rybak, K. Husain, C. Whitworth, and S. M. Somani, "Dose dependent protection by lipoic acid against cisplatin-induced ototoxicity in rats: antioxidant defense system," Toxicological Sciences, vol. 47, no. 2, pp. 195-202, 1999.

[21] T. Ishrat, M. B. Khan, M. N. Hoda et al., "Coenzyme Q10 modulates cognitive impairment against intracerebroventricular injection of streptozotocin in rats," Behavioural Brain Research, vol. 171, no. 1, pp. 9-16, 2006.

[22] R. Necker and R. F. Hellon, "Noxious thermal input from the rat tail: modulation by descending inhibitory influences," Pain, vol. 4, pp. 231-242, 1978.

[23] M. A. Ansari, S. J. Ahmad, R. Khanum, and M. Akhtar, "Pharmacological investigation of protective effects of Nigella sativa oil in experimental diabetic neuropathy in rats," Indian Journal of Pharmaceutical Education and Research, vol. 43, no. 2, pp. 166-176, 2009.

[24] S. G. Liasson, "Nerve conduction changes in experimental diabetes," Journal of Clinical Investigation, vol. 43, no. 12, pp. 2353-2358, 1964.

[25] V. Tiwari, A. Kuhad, and K. Chopra, "Tocotrienol ameliorates behavioral and biochemical alterations in the rat model of alcoholic neuropathy," Pain, vol. 145, no. 1-2, pp. 129-135, 2009.

[26] Y. Jiang, C. Guo, M. R. Vasko, and M. R. Kelley, "Implications of apurinic/apyrimidinic endonuclease in reactive oxygen signaling response after cisplatin treatment of dorsal root ganglion neurons," Cancer Research, vol. 68, no. 15, pp. 6425-6434, 2008.

[27] R. Van Doorn, C.-M. Leijdekkers, and P. T. Henderson, "Synergistic effects of phorone on the hepatotoxicity of bromobenzene and paracetamol in mice," Toxicology, vol. 11, no. 3, pp. 225-233, 1978.

[28] H. Aebi, "Catalase in vitro," Methods in Enzymology, vol. 105, pp. 121-126, 1984.

[29] J. M. Garcia, J. P. Cata, P. M. Dougherty, and R. G. Smith, "Ghrelin prevents cisplatin-induced mechanical hyperalgesia and cachexia," Endocrinology, vol. 149, no. 2, pp. 455-460, 2008.

[30] A. Kumar and S. S. Sharma, "NF- $\kappa$ B inhibitory action of resveratrol: a probable mechanism of neuroprotection in experimental diabetic neuropathy," Biochemical and Biophysical Research Communications, vol. 394, no. 2, pp. 360-365, 2010.

[31] G. Melli, M. Taiana, F. Camozzi et al., "Alpha-lipoic acid prevents mitochondrial damage and neurotoxicity in experimental 
chemotherapy neuropathy," Experimental Neurology, vol. 214, no. 2, pp. 276-284, 2008.

[32] F. L. Crane, Y. Hatefi, and R. L. Lester C Widmer, "Isolation of a quinone from beef heart mitochondria," Biochimica et Biophysica Acta, vol. 25, no. 1, pp. 220-221, 1957.

[33] F. L. Crane, "Biochemical functions of coenzyme Q10," Journal of the American College of Nutrition, vol. 20, no. 6, pp. 591-598, 2001. 

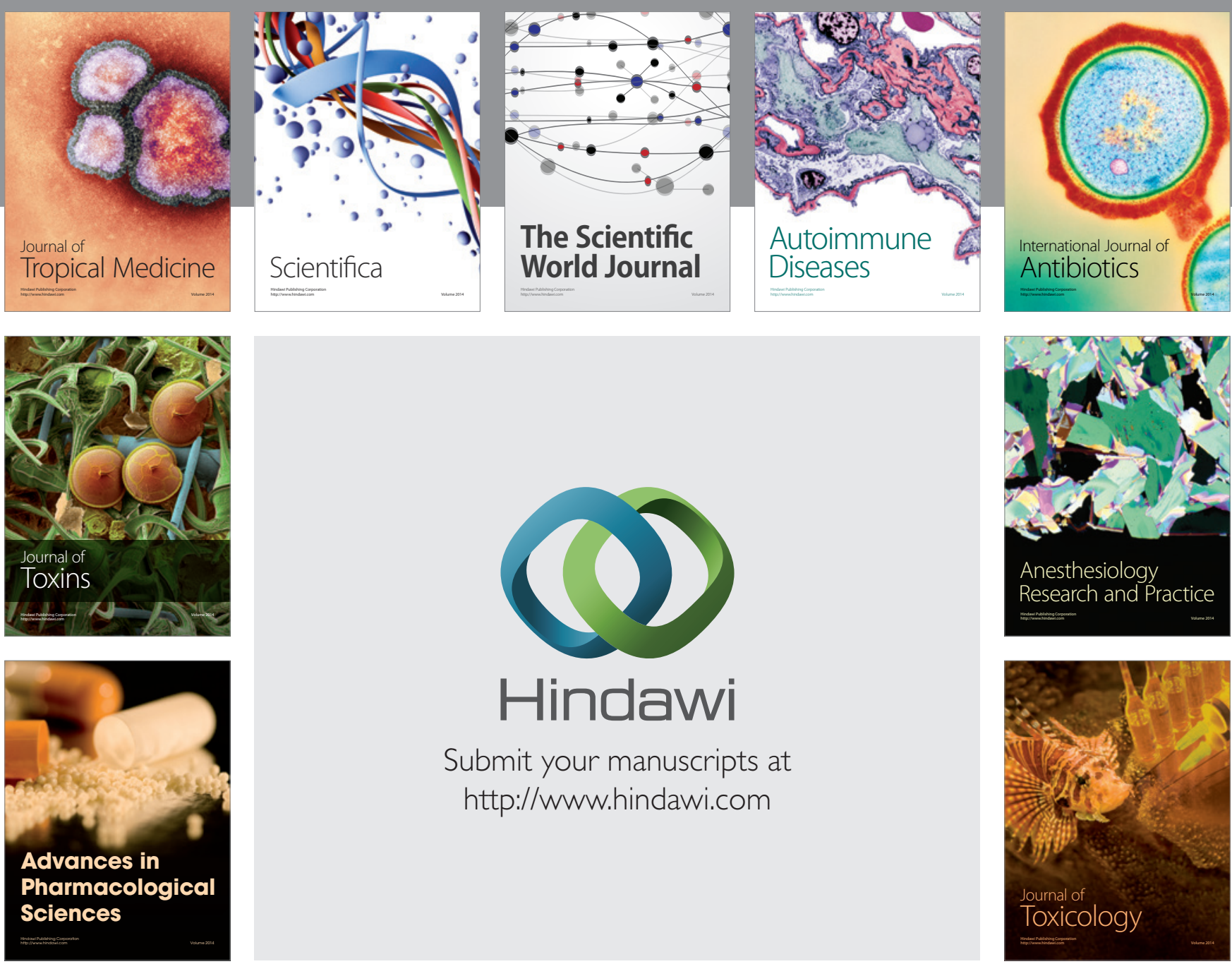

\section{Hindawi}

Submit your manuscripts at

http://www.hindawi.com
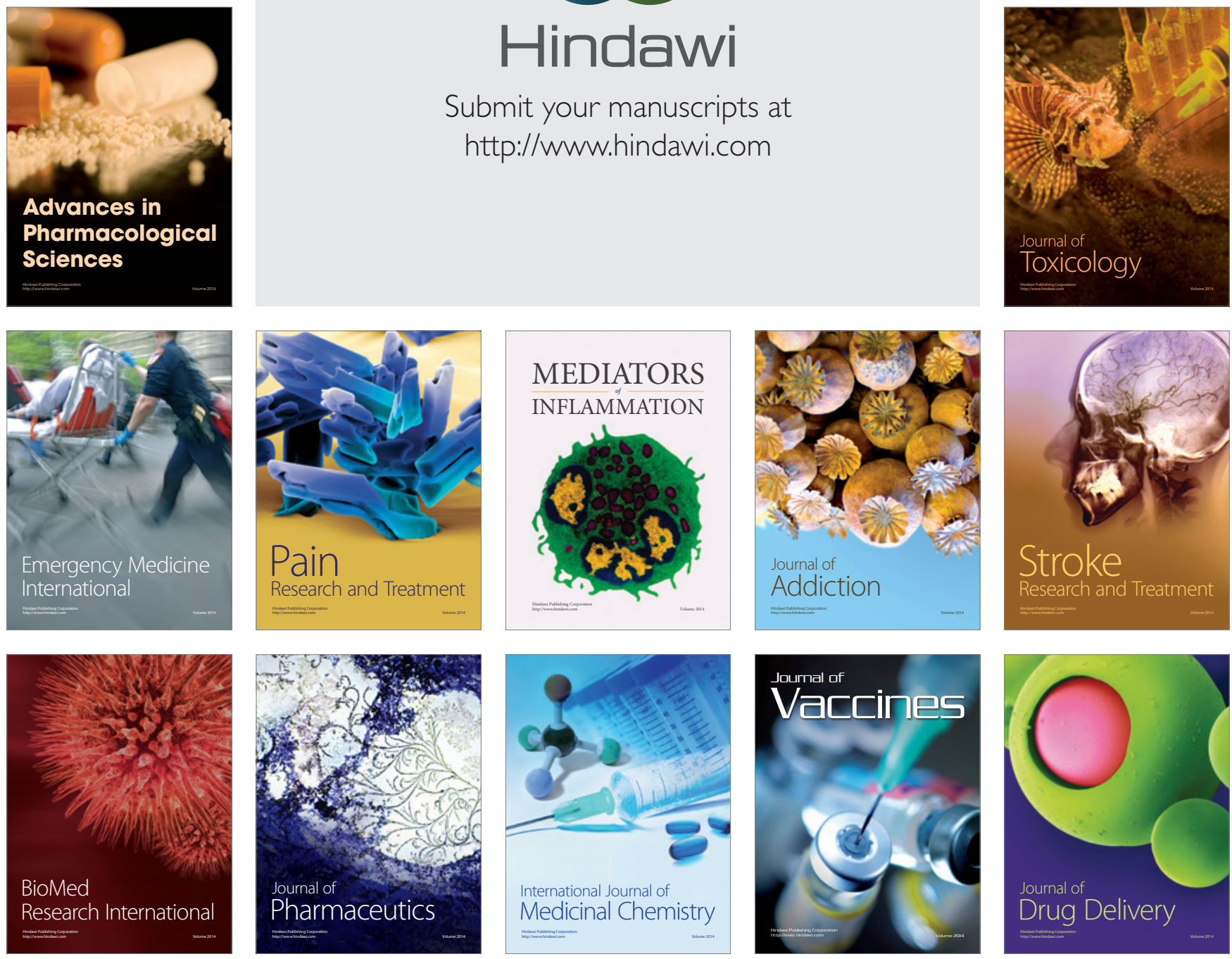